before, with total loss of speech for eight weeks, when he suddenly recovered his former relatively copious vocabulary. Neurological signs were absent, but the E.E.G. showed paroxysmal dysrhythmia. This child on follow-up also showed mental retardation and a change in character, having become erratic and incalculable in his behaviour.

His last case was of a-9-year-old boy who had sustained severe burns at 18 months. After satisfactory progress under treatment until the twentieth day convulsions appeared followed by left-sided paresis, which was still present on follow-up. There was no mental or educational retardation, though his disability caused some emotional difficulties in his relations to his peers. The E.E.G. showed a slight centro-temporal dysrhythmia.

The pathogenesis of these encephalopathies remains obscure. Although in most cases the burns appear to have been severe they were not always so. Thus in six of J. L. Emery and D. A. Campbell Reid's ${ }^{10}$ cases the burns were trivial and there was no evidence of shock in any. Sevitt argues cogently against the view that toxaemia causes the condition ${ }^{312}$; N. Roth $^{4}$ does likewise on the grounds of the time lag between the accident and the appearance of the cerebral symptoms, and so also does $\mathrm{H}$. Harbauer, ${ }^{11}$ who thinks cerebral oligaemia may be the cause. He cites in support of this the evidence of G. Ule and H. Doose. ${ }^{9}$ They report a thoroughly investigated case of a boy of 6 who sustained severe scalds at the age of 3 , followed by an encephalopathy, with residual extrapyramidal signs and severe mental retardation and who later succumbed to a catarrhal infection. Necropsy showed degenerative changes in the ganglion cells of the cortex and striatum corresponding to those found in cases of generalized oligaemia of the brain. Moreover, scarring found in the dentate nucleus and globus pallidus showed relationships to the pattern found after acute hypoxaemia-for example, in carbon monoxide poisoning.

Raised vascular and intracranial pressure has been noted in cases of burns ${ }^{12}$ and cited as a possibly pathogenetic factor. ${ }^{13}$ Cerebral oedema is considered by many authors ${ }^{213-15}$ as the cause of the cerebral degenerative changes. Sevitt ${ }^{1}$ argues against this, doubting among other things whether cerebral anoxia alone can be held responsible for the oedema. None the less the post-mortem findings, including sometimes medullary coning and other evidence of oedema, cannot be brushed aside.

It is relevant in this context to consider the electrolyte composition of the fluid transfused into the burned patients. J. Metcoff, ${ }^{16}$ on the basis of recent metabolic studies which show an unusually large loss of potassium in excess of nitrogen in burned patients, condemns the routine administration of fluids with an excess of protein, sodium, and chloride, which are retained, thus increasing oedema through over-hydration. The composition of the fluid should be adjusted to the particular needs of the individual patient, though Emery and Reid ${ }^{10}$ emphasize that their patients were in electrolyte balance at the time the cerebral symptoms appeared. No doubt an understanding of the pathogenesis of these conditions will have to await a fuller knowledge of the pathology of burns in general.

Harbauer ${ }^{11}$ notes that the majority of the children suffering from burns discharged from his clinic were more difficult, more aggressive, and more anxious than usual, and relationships with them were disturbed. This, he remarks, was not surprising in view of the long treatment with its severe pain and discomfort. Similar changes were noted by J. Woodward ${ }^{17}$ and R. T. Long and $\mathrm{O}$. Cope, ${ }^{18}$ and neurotic disturbances in adults by S. Cobb and E. Lindemann. ${ }^{19}$ Clearly these children need particularly sympathetic understanding from all who care for them, while J. M. Woodward ${ }^{20}$ has recently emphasized the value of frequent visits by the parents. Child psychiatrists too may have a part to play in preventing abnormal emotional reactions.

\section{PREVENTION OF MYOCARDIAL INFARCTION}

Occlusive vascular disease of the heart and brain is the most common cause of death in the United Kingdom. In 1961 over 23,000 men between the ages of 30 and 64 died of coronary disease in this country, about a quarter of all the deaths in this age group. ${ }^{1}$ The treatment of established myocardial and cerebral infarcts is not very effective, and it is only to be expected that the thoughts of both doctors and patients are turning to prevention of these conditions. Some patients seek advice after the death of relatives from myocardial infarction or high blood-pressure; others may bring up the topic during routine medical examinations. If the patient is fit, the first reaction is to reassure him and perhaps to advise a life of moderation and avoidance of anxiety as the best recipe for longevity. But is there any better advice than this, particularly for the patient with a bad family history or mild hypertension?

Several factors may take part in causing myocardial or cerebral infarction; these are hypertension, obesity, smoking, diabetes, and a raised serum cholesterol. Of these, the serum cholesterol and its relationship to fat in the diet, both total quantity and degree of unsaturation, have attracted most attention in the last few years. People with a moderately raised serum cholesterol have 
a greater risk of myocardial infarction than those with a lower one. One of the most convincing illustrations of this has come from the prospective study conducted in the town of Framingham, Massachusetts. ${ }^{2}$ In this town a group of people with a serum cholesterol of over $244 \mathrm{mg}$. per $100 \mathrm{ml}$. had a three times higher incidence of coronary heart disease than a companion group with a cholesterol of less than $210 \mathrm{mg}$. per $100 \mathrm{ml}$. But though the level of serum cholesterol may be lowered by diet or drugs there is no convincing evidence that to do this improves the prognosis. ${ }^{3}$

Hypertension, obesity, and diabetes are the well-known trio that attract the interest of actuaries concerned with the terms of life policies. Of these, hypertension is the most common and probably the most sinister. It is not always appreciated that comparatively small increases of the blood-pressure are associated with a considerable rise in mortality. The American Society of Actuaries ${ }^{4}$ found that men under 50 with a bloodpressure of about $170 / 100 \mathrm{~mm}$. had three and a half times the mortality of men whose blood-pressure was within normal limits. Older men fared better, but a pressure at this level in the age range 50-59 still more than doubled the mortality ratio. Even a blood-pressure of $150 / 90 \mathrm{~mm}$. nearly doubled the mortality ratio from all causes in men under 50. Most of the excess deaths were caused by vascular disease of the brain, heart, or kidneys, and deaths from coronary-artery disease were twice as common as in men with normal blood-pressure. Mild hypertension of this order is not usually taken very seriously except in patients under 40 , and is rarely treated with drugs: there is no really convincing evidence for or against such treatment as a prophylactic measure. If treatment were completely effective in eliminating the adverse effects of mild hypertension, enormous numbers of patients would have to be treated for years to save a small proportion of them from early death. It is a daunting prospect, and at present there is no suggestion of any tolerable or practical way to reduce the incidence of mild hypertension in the community.

Obesity attracts attention because in theory it should be easier to prevent than hypertension and raised serum cholesterol. But in practice fat people often seem more willing to take pills than to eat less. The obese may derive some comfort from the fact that being overweight by itself is not so dangerous as having mild hypertension." A man in the age range 15-69 who is $20 \%$ (say. $35 \mathrm{lb}$. or $16 \mathrm{~kg}$.) overweight has only a $20 \%$

1 Smoking and Health, A Report of the Royal College of Physicians, 1962. London. Kannel, W. B.. et al.. Ann. intern. Med. 1961, 55, 33. Oliver, M. S.. and Boyd, G. S., Lancet, 1961, 2, 499.

Society of Actuaries. Build and Blood Pressure Study, 1959, Chicago.

Hammond. E. C., and Horn, D.. J. Amer. med. Ass. 1958, 166, 1294.

?Doll. R.. and Hill. A. B., B-it. med. J., 1956, $2,1071$.

- Wld Hith Org. techn. Rep. Ser., No. $23 \mathrm{i}$, "Arterial Hypertension and Ischaemic Heart Disease-Preventive Aspects," 1962, Geneva. increase in the expected mortality from all causes, though if he is 35 to $40 \%$ overweight the mortality ratio is raised about $50 \%$. Mortality from diabetes shows a sharper upward trend than any other cause with increasing weight, but mortality from coronary disease is roughly double in the very obese what it is in men of average build. Diabetes itself is associated with an increased risk of vascular occlusion. Improved methods of detection may improve the prognosis of diabetics, but control of hyperglycaemia does not necessarily prevent vascular disease.

The plume of cigarette smoke on the health-education poster spells out "Cancer," but it might very well spell out "Coronary" as well. Bronchial carcinoma has naturally attracted public attention, but surveys of mortality in relation to smoking habits have revealed a much larger excess mortality among smokers than can be accounted for by cancer. E. C. Hammond and D. Horn $^{5}$ found that smokers had a $70 \%$ higher death rate from coronary disease than non-smokers, and R. Doll and $\mathrm{A}$. Bradford $\mathrm{Hill}^{6}$ found a fourfold higher death rate from coronary disease among doctors under 55 years old who smoked more than 25 cigarettes daily. In the Framingham survey the death rate from coronary disease among middle-aged heavy smokers was nearly five times that of non-smokers.

Many would therefore agree with a recent Expert Committee of the World Health Organization which concluded that there were no effective means of preventing ischaemic heart disease. ${ }^{8}$ But since there is strong evidence that smoking is partly responsible for causing ischaemic heart disease, particularly in middle age, patients should be advised to give it up. Apart from that there is little that need be done except to reassure the symptomless but worried patient. The examination of him should of course have included weighing him and testing the urine for sugar. If the family history of myocardial infarctions is exceptionally bad, particularly among young adults, more thorough investigation is desirable because of the possibility of familial hypercholesterolaemia.

\section{PRESIDENT KENNEDY}

The tragedy of President Kennedy's assassination has struck with special force at the hearts of medical men and women in Britain. His devotion to improving the welfare of people enfeebled by age or mental infirmity was, like much else of his work, courageous, imaginative, and humane. Early in his term of office President Kennedy made the care of mental patients a matter of his personal concern, calling in words so characteristic of the man for a "bold new approach" to the provision 